\title{
Sélection du palmier à huile pour une huile de palme durable et responsabilité sociale
}

\author{
Tristan DURAND-GASSELIN ${ }^{1}$ \\ Lisa BLANGY ${ }^{1}$ \\ Christian PICASSO ${ }^{1}$ \\ Hubert DE FRANQUEVILLE ${ }^{2}$ \\ Frederic BRETON ${ }^{2}$ \\ Philippe AMBLARD \\ Benoit COCHARD ${ }^{2}$ \\ Claude LOUISE ${ }^{1}$ \\ Bruno NOUY \\ 1 Batiment 14, \\ Parc Agropolis, 2214, boulevard de la Lironde, \\ 34980 Montferrier sur Lez, \\ France \\ <tristan.durand-gasselin@palmelit.com> \\ 2 UPR 28 \\ Amélioration génétique du palmier à huile Cirad, \\ Bios TA 28/01, avenue Agropolis, \\ F-34398 Montpellier Cedex 05, \\ France
}

La sélection des plantes est une histoire ancienne, si longue, 10000 ans environ, que I'on recherche même des marqueurs génétiques de domestication des plantes. Elle recèle cependant une pratique très variable selon les espèces, et il est très difficile de distinguer ce qui relève d'une cueillette organisée de véritables actes de sélection. Chez le palmier à huile, on peut trouver quelques plantations réalisées pour répondre à des besoins commerciaux au XIX ${ }^{\mathrm{e}}$ siècle : le roi d'Abomey Guézo (1818-1858) développa des plantations dans le but de maintenir les ressources de l'État, privées du commerce des esclaves. Ces plantations étaient sans doute encouragées par de nombreuses missions commerciales effectuées par les Européens entre 1840 et 1860 (Jannot, 2001). On rencontre encore en Côte-d'Ivoire des plantations plantées réalisées à la fin de ce siècle avec des palmiers prélevés dans les palmeraies naturelles (figure 1).

À cette époque, il s'agissait surtout de cueillette organisée. Ce n'est qu'un peu plus tard, et en Asie, que les premiers actes de sélection véritable ont été réalisés. Ils ont conduit au développement d'une population de palmiers,

Abstract: As an actor of a supply chain, seed producers must take into account the impact of their work on sustainability. This is particularly true when one works on palm oil, a highly sensitive crop which is questioned on its social and environmental impact. Breeders act directly on yield thus increasing profitability, while for the same production, decreasing land-use pressure. In addition, breeders act on duration of the plantations - which is critical for a perennial crop - making strategic choices selecting for lasting resistances to diseases. They can seek rustic selections, which help the work of small holders and enable them to a better social insertion in the supply chain. In the long trend, the promotion of sustainable palm oil is an excellent opportunity for palm oil seed distributors. Corporate and Social Responsibility of PalmElit, company recently created, is presented. It defines its aspirations, its values, its direct and indirect responsibilities and finally proposes an operational code of conduct.

Key words: oil palm, breeding, disease resistance, corporate social responsibility, CSR, code of conduct, sustainability

appelée «Deli » du nom d'une province de nord Sumatra, extrêmement importante pour la sélection du palmier à huile (Lubis, 1988).

Nous avions rappelé les principales étapes de la sélection du palmier à huile en 2002 (DurandGasselin et al., 2002). Dès 2005, nous nous étions interrogés sur l'impact de la sélection du palmier à huile sur la durabilité des plantations (Cochard et al., 2005). Nous n'en rappellerons ici que les principaux aspects en soulignant les objectifs poursuivis et leur impact technique sur la durabilité des plantations.

En 2009, le Cirad a décidé de créer une filiale, PalmElit SAS, chargée de maintenir et d'accrô̂tre ses recherches de création de variétés de palmier à huile ainsi que de promouvoir leur diffusion au plus grand nombre. Cela a été I'occasion pour le Cirad et sa filiale de s'interroger sur la meilleure façon de conduire cette activité dans un cadre de responsabilité sociale et entrepreneuriale (RSE). Un document, définissant la stratégie RSE de l'entreprise, a été élaboré puis traduit en un code de conduite. Nous retracerons les principales interrogations auxquelles nous pensons devoir faire face. Cet exercice a également permis de souligner l'importance de l'initiative RSPO (www.rspo. org) dont le succès et la généralisation à l'ensemble de l'industrie du palmier à huile seront une base indispensable au développement d'une filière palmier à huile durable.

\section{Sélection pour une culture durable}

\section{En quoi le sélectionneur tient-il compte} de la durabilité?

S'interroger sur sa pratique scientifique devrait sans aucun doute être un des aspects les plus importants du travail d'un chercheur: il doit, en effet, participer à la définition des objectifs qui peuvent, ou non, être en adéquation avec les attentes de la société. Sans doute doit-il être aidé dans sa réflexion par l'émergence d'une conscience largement partagée, prisme à travers lequel il revisitera son travail et s'efforcera de prendre en compte les enjeux sociétaux. Le développement durable est clairement un enjeu planétaire qui se formalise puis se décline dans un grand nombre de domaines. 


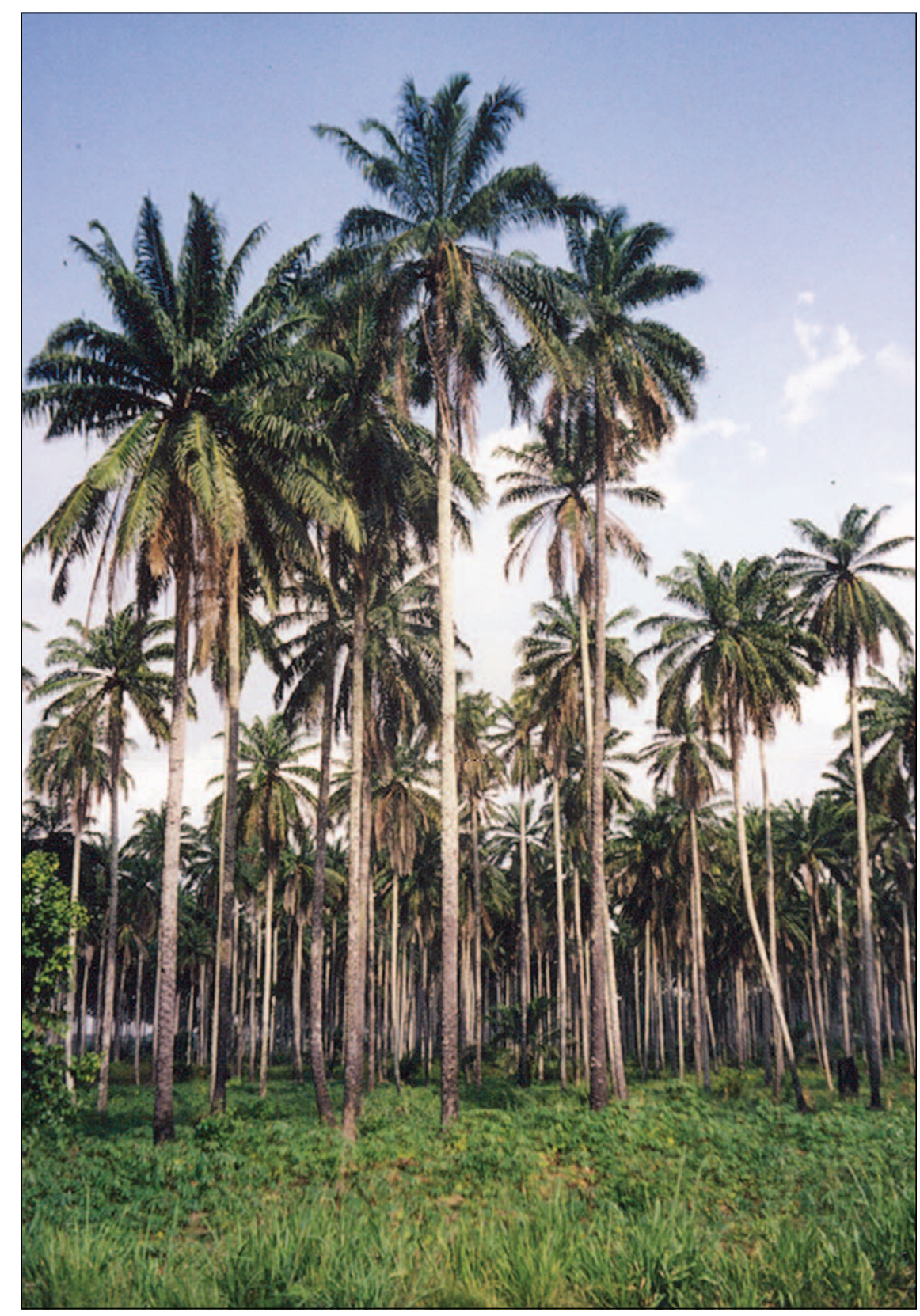

Figure 1. Palmeraie naturelle, route de Dabou (Côte-d'Ivoire), plantée vers 1880. (Photo T. Durand-Gasselin).

Comment notre métier de sélectionneur s'inscrit-il dans ce cadre? Pour beaucoup $d^{\prime}$ entre nous qui travaillons le palmier à huile, il n'y a là rien de bien neuf, habitués que nous sommes, sélectionneurs de plante pérenne, aux projections longues et même très longues, et confrontés par nécessité et depuis l'origine à la durée. La durabilité va pourtant au-delà de la simple durée avec la recherche d'une activité viable (" sustainable ») dans laquelle on entend mieux la connotation économique et sociale, le bien-être du cultivateur de palmier à huile, mais également son interaction avec le monde audelà de sa plantation : son insertion écologique plus que les rendements eux aussi sont en progression. La relation entre la qualité du matériel végétal et l'équité sociale est sans doute plus difficile à concevoir, mais doit être discutée : le type de matériel végétal peut-il influencer le modèle de développement paysan ou même agro-industriel ?

Cependant, il ne faut pas attendre des généticiens de solutions à toutes les attentes de la filière et à tous les impacts négatifs du palmier à huile. Pour commencer, le progrès génétique sur les rendements reste insuffisant, même pour les meilleurs obtenteurs (1 à 1,5\% par année), pour répondre à l'augmentation des besoins en huile végétale (+3 à $4 \%$ ) en général et celle en huile de palme en particulier ( +5 à $6 \%$ ) (figure 2).

La généralisation de bonnes pratiques agronomiques et la gestion intégrée des maladies (à laquelle la génétique participe) sont donc également des volets très importants. Enfin, si le rythme de développement de la consommation d'huile végétale continue à la même vitesse, il sera inévitable d'augmenter encore les surfaces cultivées et, pour cela, on devrait privilégier la réhabilitation de terrains dégradés ou de pâtures. Les tableaux 1 et 2 donnent cependant une idée du potentiel formidable du palmier à huile pour couvrir ces besoins avec un impact écologique comparativement bien plus faible que les autres oléagineux, à condition de bien choisir les zones de développement, ce qui est du ressort des États qui doivent organiser et contrôler ce développement. Par exemple, en près de 20 ans on aura produit 34,5 millions de tonnes d'huile de palme supplémentaires pour une surface cultivée supplémentaire de 8,55 millions d'hectares contre 20 millions de tonnes d'huile de soja, le tourteau en plus également, pour 48 millions d'hectares supplémentaires et 13 millions de tonnes d'huile de colza (plus le tourteau) pour 13 millions d'hectares. Les progrès sur les rendements sont aussi bien différents (respectivement $+24 \%,+20 \%$ et $+43 \%$ ).

La prise en compte de nouveaux critères de sélection est un processus long et difficile. Après s'être assuré que le nouveau critère de sélection envisagé restera valide sur le long terme, il faut éventuellement définir les outils de sélection (comment mesurer ce caractère), connaître la diversité génétique disponible, étudier l'héritabilité du caractère et son impact éventuel sur les méthodes de sélection. Sans doute faut-il également mesurer un ratio bénéfice attendu au regard des efforts fournis.

\section{Sélection pour le rendement}

Dans notre article précédent, nous avions distingué trois grandes périodes de l'amélioration génétique pour le rendement (Durand- 
Tableau 1. Évolution des surfaces plantées (x 000 ha) pour les principales plantes oléagineuses dans le monde (source Oilworld).

\begin{tabular}{|llllll|}
\hline & $\mathbf{1 9 9 0}$ & $\mathbf{1 9 9 5}$ & $\mathbf{2 0 0 0}$ & $\mathbf{2 0 0 5}$ & $\mathbf{2 0 0 9}$ \\
\hline Palmier à huile $^{\mathrm{a}}$ & 3560 & 4690 & 6560 & 9187 & 12117 \\
\hline Soja & 54910 & 61960 & 75260 & 92813 & 102400 \\
\hline Tournesol & 16200 & 20230 & 19740 & 23207 & 23810 \\
\hline Colza & 17790 & 24520 & 25260 & 27371 & 31030 \\
\hline Coton & 33920 & 35150 & 32210 & 34251 & 30900 \\
\hline Arachide & 19910 & 20590 & 22560 & 22523 & 20910 \\
\hline Cocotier & 8940 & 9210 & 9570 & 9270 & 9540 \\
\hline
\end{tabular}

${ }^{\text {a }}$ Surfaces en production.

Tableau 2. Évolution des productions et des rendements à l'hectare pour les principales plantes oléagineuses dans le monde (source Oilworld).

\begin{tabular}{|c|c|c|c|c|c|c|c|c|c|c|}
\hline & \multicolumn{5}{|c|}{ Production d'huile (tonnes $\times 1000$ ) } & \multicolumn{5}{|c|}{ Rendement en huile (tonnes/ha) } \\
\hline & 1990 & 1995 & 2000 & 2005 & 2009 & 1990 & 1995 & 2000 & 2005 & 2009 \\
\hline Huile de palme & 10710 & 15201 & 21861 & 33847 & 45263 & 3,01 & 3,24 & 3,33 & 3,68 & 3,74 \\
\hline Soja & 15760 & 20231 & 25562 & 33567 & 36016 & 0,29 & 0,33 & 0,34 & 0,36 & 0,35 \\
\hline Tournesol & 7900 & 8635 & 9743 & 9821 & 13099 & 0,49 & 0,43 & 0,49 & 0,42 & 0,55 \\
\hline Colza & 8720 & 10631 & 14500 & 16292 & 21604 & 0,49 & 0,43 & 0,57 & 0,60 & 0,70 \\
\hline Coton & 3860 & 3875 & 2850 & 5007 & 4714 & 0,11 & 0,11 & 0,12 & 0,15 & 0,15 \\
\hline Arachide & 3800 & 4252 & 4539 & 4513 & 4208 & 0,19 & 0,21 & 0,2 & 0,20 & 0,20 \\
\hline Cocotier & 8940 & 9210 & 9570 & 3255 & 3226 & 0,34 & 0,31 & 0,34 & 0,35 & 0,34 \\
\hline
\end{tabular}

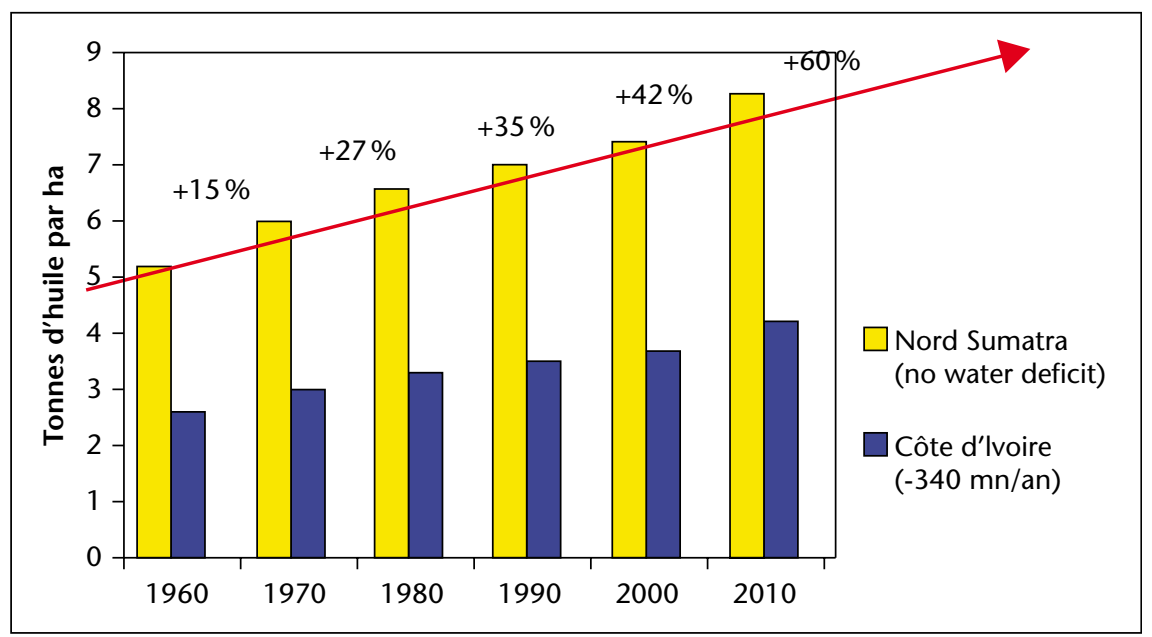

Figure 2. Représentation du progrès génétique réalisé au cours des cycles de sélection du palmier à huile : valeur génétique des semences commerciales en Indonésie (pas de déficit hydrique) et en Côte-d'lvoire (340 mm de déficit hydrique).

Gasselin et al., 2002). Dans l'entre-guerre, tant en Afrique qu'en Asie du Sud-Est, les centres de recherche ont mené des sélections massales qui ont conduit à quelques progrès lents, mais significatifs surtout en Asie où l'absence de dis- ont été rapidement adoptées par l'industrie, car elles engendraient un progrès de plus de $30 \%$ pour la production d'huile. La troisième phase débute pour les chercheurs dans les années 1950 lorsqu'un effet d'hétérosis important est mis en évidence entre les populations africaines et la population Deli (Gascon et de Berchoux, 1964). Immédiatement, presque $10 \%$ supplémentaires ont été gagnés.

L'adoption, au moins par une partie des centres de recherche pour l'amélioration génétique du palmier à huile, d'un schéma de sélection récurrente réciproque, dont un des fondements est l'exploitation de cet hétérosis, permet aujour$d$ 'hui encore un progrès continu de la valeur des semences i (figure 2) (Durand-Gasselin et al., 2000).

Chaque cycle de sélection, qui dure une vingtaine d'année en pratique, permet une augmentation de la production de $15 \%$ environ (Gascon et al., 1981 ; Gascon et al., 1988 ; Cochard et al., 1993).

Ces progrès génétiques ont eu un impact réel sur les rendements en plantation et, en Malaisie, un exemple d'augmentation du rendement par hectare de 1,3 à 5,4 tonnes d'huile, dont la moitié est attribuée à la génétique est cité par Davidson (1993). En Indonésie, une plantation commerciale dont le périmètre n'a pas été modifié depuis longtemps voit ses rendements augmenter régulièrement, illustrant ainsi sa capacité à tirer parti des progrès génétiques réalisés, même si les nouveaux matériels sont utilisés en replantation sur des terrains moins fertiles que ceux d'une première génération (figure 3).

À ce jour, les populations africaines n'ont subi que deux à trois véritables générations d'amélioration. La population Deli est, elle, un peu plus avancée. Mais tout cela reste très faible au regard du travail réalisé pour des plantes annuelles. Néanmoins, depuis 50 ans, on constate que l'amélioration génétique, stricto sensu apporte un progrès continu estimé à $1 \%$ par an (Hardon et al., 1987, Durand-Gasselin et al., 2000) et il est possible d'envisager les 10 tonnes $d^{\prime}$ 'huile/hectare par an (Durand-Gasselin et al., 2003).

Malheureusement, bien souvent, ce progrès génétique n'est pas disponible pour les petits paysans qui n'ont souvent accès qu'à du matériel non sélectionné dit " tout venant ${ }^{1}$ ", pour lequel les rendements sont inférieurs de $60 \%$ à ceux du matériel amélioré (Cochard et al., 2001).

onction pour l'épaisseur de la coque rendait sélection plus facile. La découverte de I'hérédité de l'épaisseur de la coque (Beirnaert et Vanderweyen, 1941) a permis la production de semences $100 \%$ tenera (voir Encadré), qui

\footnotetext{
${ }^{1}$ Matériel dit « tout venant » : graines récoltées dans les plantations existantes.
} 


\section{Encadré 1}

Hérédité de l'épaisseur de la coque du fruit de palmier à huile

L'épaisseur de la coque est contrôlée par un gène codominant (sh). Les palmiers pisifera, homozygote sh- (absence de coque), ont le fruit le plus riche en pulpe et donc en huile, mais présentent une très forte stérilité femelle et sont donc peu productifs. Les arbres à type de fruit dura, homozygote sh+ ont une coque épaisse alors que les hétérozygotes appelés tenera (issus, par exemple, du croisement de palmiers dura par des palmiers pisifera) ont une coque mince et donc plus de pulpe. Pour cette raison, les dura produisent moins d'huile que les tenera. Ces derniers sont donc les plus intéressants en plantation (+33\% en huile par rapport aux dura).

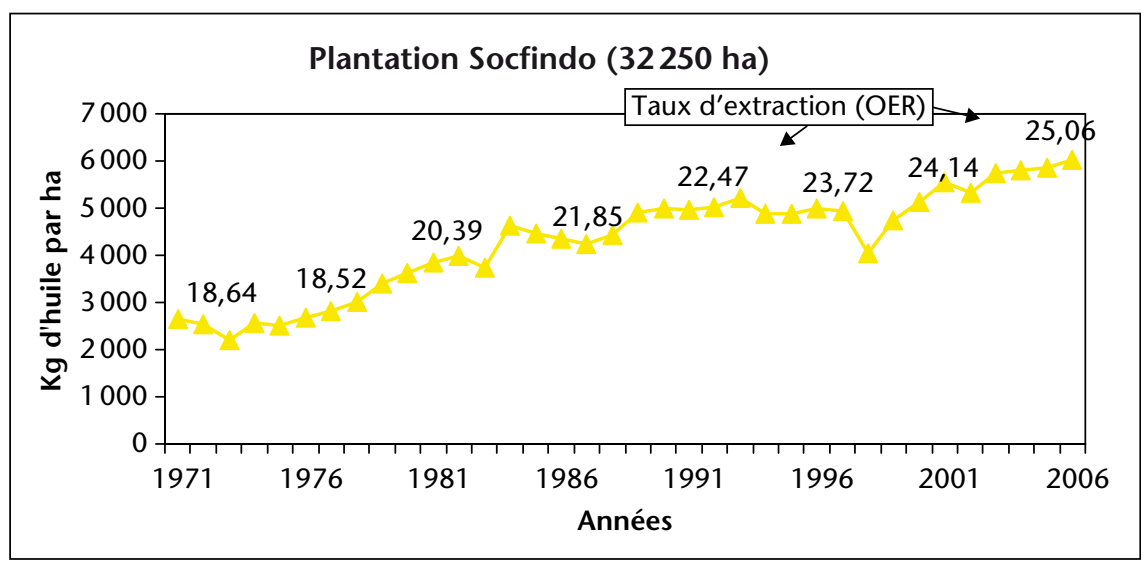

Figure 3. Représentation de l'évolution des rendements des plantations de la Socfindo (Indonésie). On note l'impact de la crise économique de 1998 marquée par des vols importants.

\section{Sélection pour la résistance aux maladies chez le palmier à huile}

Le palmier à huile est sensible à trois maladies qui ont une importance économique majeure. Un Fusarium (Fusarium oxysporum fs elaeidis), assez spécifique à l'Afrique, peut provoquer des pertes extrêmement importantes en plantation (jusqu'à $70 \%$ ) (de Franqueville et Renard, 1990). En Asie du Sud-Est, le Ganoderma a pu provoquer jusqu'à $80 \%$ de mortalité, mais cette maladie est également largement présente en Afrique, parfois en combinaison avec la fusariose, et des foyers apparaissent aujourd'hui en Amérique latine (de Franqueville et al., 2001). Enfin, en Amérique latine sévit une pourriture du cœur, probablement liée à Phytophthora palmivora (mais il convient encore de confirmer s'il est en interaction avec d'autres pathogènes), qui provoque jusqu'à $100 \%$ de mortalité (de Franqueville, 2003; Martinez et al., 2008). Pour ces

\section{Encadré 2 \\ À propos de résistance et de tolérance}

Les phytopathologistes et les sélectionneurs font une distinction claire entre les résistances totales (qui sont spécifiques) et les résistances partielles (non spécifiques). Les résistances totales proviennent généralement d'une interaction gène à gène entre la plante et le pathogène. La résistance gène à gène est largement utilisée par les sélectionneurs. Toutefois de telles résistances spécifiques sont souvent contournées par le pathogène: pour le palmier à huile, comme pour les plantes pérennes en général, nous ne devons pas rechercher de telles résistances. La stratégie à privilégier est de sélectionner de multiples gènes impliqués dans des mécanismes de défense pour conférer à la plante des résistances partielles au pathogène. Une telle sélection favorisera l'acquisition d'une résistance durable, utile face à une large diversité d'isolats (non spécifiques). Sélectionner ainsi des résistances partielles ne conduira pas à la disparition de plants malades dans les champs mais limitera leur fréquence.

Les agronomes et les planteurs utiliseront plus souvent le terme de « tolérant » pour désigner les variétés porteuses de multiples (ou somme de) résistances partielles. trois maladies, des résistances génétiques ont été identifiées et sont déjà intégrées dans des variétés « résistantes » disponibles pour les plantations, ou le seront à court terme.

II s'agit là de caractéristiques qui ont une influence majeure sur la durabilité des cultures. La résistance aux maladies sécurise l'investissement réalisé et participe à garantir les profits à long terme.

\section{Fusariose}

Suite à un long travail, les sélectionneurs ont mis à disposition des planteurs du matériel hautement résistant et hautement productif qui tire parti des sources de résistances identifiées en plantation dans les années 1960 (Renard et al., 1980 ; Renard et Meunier, 1983; Durand-Gasselin et al., 2000).

La figure 4 illustre comment, dans une plantation très fortement affectée par la fusariose, l'incidence de cette maladie a peu à peu décliné au fil des ans en raison de la plantation de matériel de plus en plus résistant.

Du matériel fortement résistant et hautement productif est disponible pour l'ensemble des plantations réalisées en Afrique. Aujourd'hui, nos recherches portent sur l'exploitation de nouvelles sources de résistances de façon à élargir la base du matériel résistant. Nous travaillons également à la recherche de matériel présentant une double résistance à la fusariose et au Ganoderma.

\section{Ganoderma}

Le travail de recherche de variétés résistantes au Ganoderma est bien plus récent que pour la fusariose (Breton et al., 2009). Il n'a pas abouti encore à la diffusion de variétés résistantes au Ganoderma, mais les résultats sont très encourageants. En champ, nous observons de grandes différences (Durand-Gasselin et al., 2005) (figure 5).

Un test précoce a ensuite été développé (Breton et al., 2009), il permet d'inoculer, dès le stade de prépépinière, des plantules et de tester très rapidement la résistance au Ganoderma de différentes origines de palmier à huile. Par exemple, dans le tableau 3, différentes origines "Afrique », toutes croisées avec la même origine Deli, sont comparées pour leur résistance au Ganoderma en prépépinière. Les différences exprimées en prépépinière sous une forte pression d'inoculation se traduisent en champ par des fréquences de maladie également variables. II n'y a pas nécessairement de lien entre la résistance à la fusariose et la résistance au Ganoderma, cependant nous avons identifié quelques génotypes qui semblent présenter une double résistance aux deux maladies.

Cette double résistance est primordiale pour I'Afrique centrale (bassin du Congo, Cameroun) où les deux maladies sont très actives; on rencontre également, mais de façon plus 


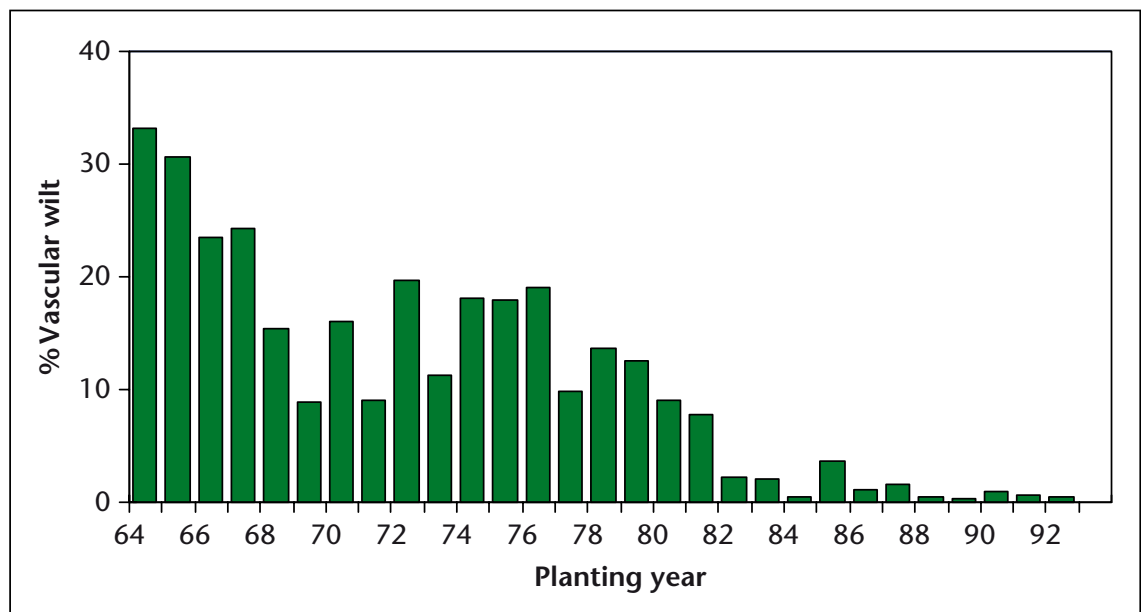

Figure 4. Évolution du pourcentage de plants atteints de fusariose dans la plantation de Robert Michaux de Dabou en Côte-d'ivoire en fonction des années de plantation.

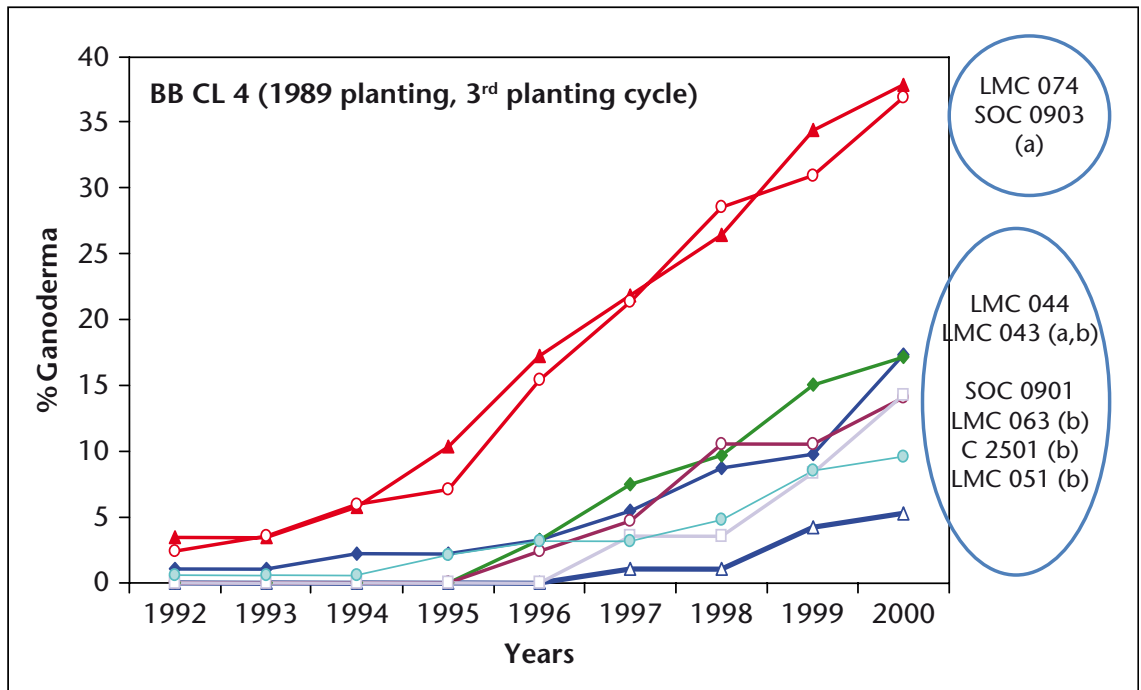

Figure 5. Évolution avec le temps du pourcentage de plants infectés par le Ganoderma dans l'essai comparatif de clones BBCL4 (plantation de Bangun Bandar, Socfindo, Indonésie).

Tableau 3. Quelques origines pisifera "Afrique" confrontées à des Deli sensibles.

\begin{tabular}{|c|c|c|c|c|}
\hline Groupe B & Groupe A & $\begin{array}{l}\text { Nombre } \\
\text { de pisifera }\end{array}$ & $\begin{array}{l}\text { Pourcentage } \\
\text { de Ganoderma } \\
\text { exprimé }\end{array}$ & $\begin{array}{l}\text { Nombre de croisements } \\
\text { testés (nombre de tests) }\end{array}$ \\
\hline Pisifera d'origine $\mathrm{A}$ & \multirow{8}{*}{$\begin{array}{l}\text { Origine Deli } \\
\text { unique }\end{array}$} & 4 & 28,2 & (11 croisements, 11 tests) \\
\hline Pisifera d'origine B & & 3 & 29,1 & (10 croisements, 11 tests) \\
\hline Pisifera d'origine C & & 3 & 30,3 & (5 croisements, 10 tests) \\
\hline Pisifera d'origine D & & 8 & 34,5 & (15 croisements, 15 tests) \\
\hline Pisifera d'origine $E$ & & 7 & 35,4 & (12 croisements, 12 tests) \\
\hline Pisifera d'origine $F$ & & 4 & 35,4 & (8 croisements, 8 tests) \\
\hline Pisifera d'origine $\mathrm{G}$ & & 14 & 37,5 & (18 croisements, 22 tests) \\
\hline Pisifera $\mathrm{d}^{\prime}$ origine $\mathrm{H}$ & & 1 & 37,5 & (11 croisements, 11 tests) \\
\hline
\end{tabular}

ponctuelle les deux maladies ensemble en Afrique de l'Ouest (de Franqueville, communication personnelle). Parmi les variétés qui possèdent ces caractéristiques, il y en existe de très productives, ce qui permettra de ne pas faire de compromis sur ce point.

\section{Pourriture de cœur}

La symptomatologie de la pourriture du cœur (PC) est complexe, très variable d'une région à I'autre. Probablement liée à Phytophthora palmivora il est possible qu'il y ait un cocktail de maladies opportunistes secondaires qui se développent à sa suite (de Franqueville, 2003 ; Martinez et al., 2008). Nous parlons parfois de complexe de maladies à PC.

On ne dispose pas aujourd'hui d'un test précoce, et le seul moyen de mettre en évidence des différences de résistance entre variétés reste le champ (Amblard et al., 2009) (figure 6). Aujourd'hui, tout indique que les résistances observées dans un environnement donné sont également valables dans un autre environnement, même si les symptômes sont parfois bien différents. On n'observe pas, chez Elaeis guineensis, de variétés fortement résistantes, même si les travaux présentés par Amblard et al. sont, de ce point de vue, très prometteurs (Amblard et al., 2009). Le tableau 4 illustre la grande variabilité observée au sein de l'origine Deli pour la résistance à la PC; ces facteurs de résistance, combinés à des sources de résistance issues des origines "Afrique », qu'il reste à identifier, permettraient de développer à terme des variétés résistantes au complexe de maladie PC.

Il a été montré que les hybrides interspécifiques entre des populations choisies d'Elaeis oleifera et des origines sélectionnées $d^{\prime} E$. guineensis présentent une très forte résistance qui, tout en n'étant pas totale, reste, aujourd'hui, la meilleure solution pour les planteurs.

Les différentes stratégies de sélection qui en résultent ont été évoquées dernièrement (Durand-Gasselin et al., 2009) et ouvrent de nombreuses perspectives qui exploiteraient les atouts $d^{\prime} E$. oleifera en combinant différentes approches : la création d'hybrides interspécifiques, l'introgression de certains caractères dans $E$. guineensis, les techniques de culture in vitro i (figure 7), en tirant et tireraient profit de travaux spécifiques sur la sélection assistée par marqueurs.

La réponse génétique apportée aujourd'hui, I'hybride interspécifique, souffre d'un handicap majeur, qu'il est intéressant de souligner dans cet article, dans la mesure où il illustre parfaitement comment les programmes de création variétale peuvent avoir une influence sur certains aspects sociaux de la culture du palmier à huile.

Aujourd'hui, le pollen produit par I'hybride interspécifique est peu fertile. De plus, les insec- 


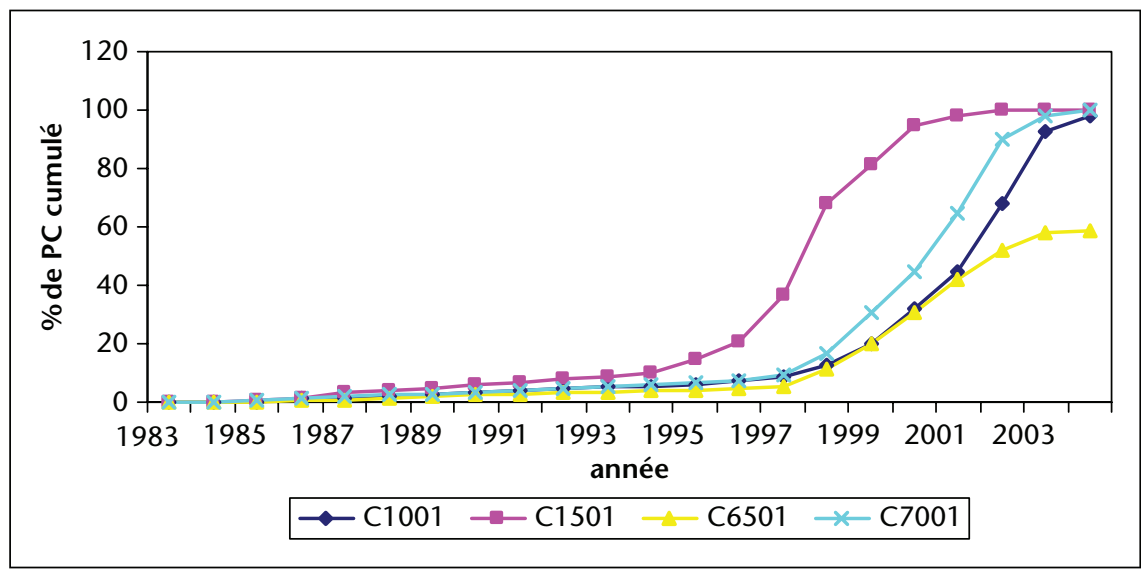

Figure 6. Évolution de la pourriture du cœur dans l'essai SHGP 1 (Plantation 1983, Shushufindi Palmeras de los Ecuador, Équateur).

Tableau 4. Pourcentage de mortalité à neuf ans par pourriture du cœeur dans les essais SHGP 9 et 10 (Plantation 2000, Shushufindi, Palmeras de los Ecuador, Équateur) par origine Deli.

\begin{tabular}{|lll|}
\hline Origine Deli & SHGP 9 & SHGP 10 \\
\hline DA 8 D AF & 68,0 & 38,1 \\
\hline LM 404 D AF & 62,0 & 37,5 \\
\hline DA 115 D × DA 3 D & 51,9 & 24,6 \\
\hline DA 5 D × DA 3 D & 48,8 & 29,6 \\
\hline DA 115 D AF & 47,8 & 29,2 \\
\hline DA 128 D AF & 19,6 & 9,5 \\
\hline
\end{tabular}

tes pollinisateurs inféodés soit à $E$. guineensis soit à E. oleifera sont peu attirés par les inflorescences de l'hybride. La pollinisation naturelle des inflorescences femelles de l'hybride reste donc très difficile et il faut réaliser une pollinisation assistée manuellement, ce qui demande une organisation pour la récolte et le conditionnement du pollen que les petits planteurs auraient du mal à réaliser seuls. Cette difficulté pourrait être un frein majeur au développement de la culture des hybrides en excluant de la chaîne de production la petite agriculture familiale. Nous pensons cependant qu'une organisation coopérative pourrait permettre de prendre en charge les infrastructures néces-

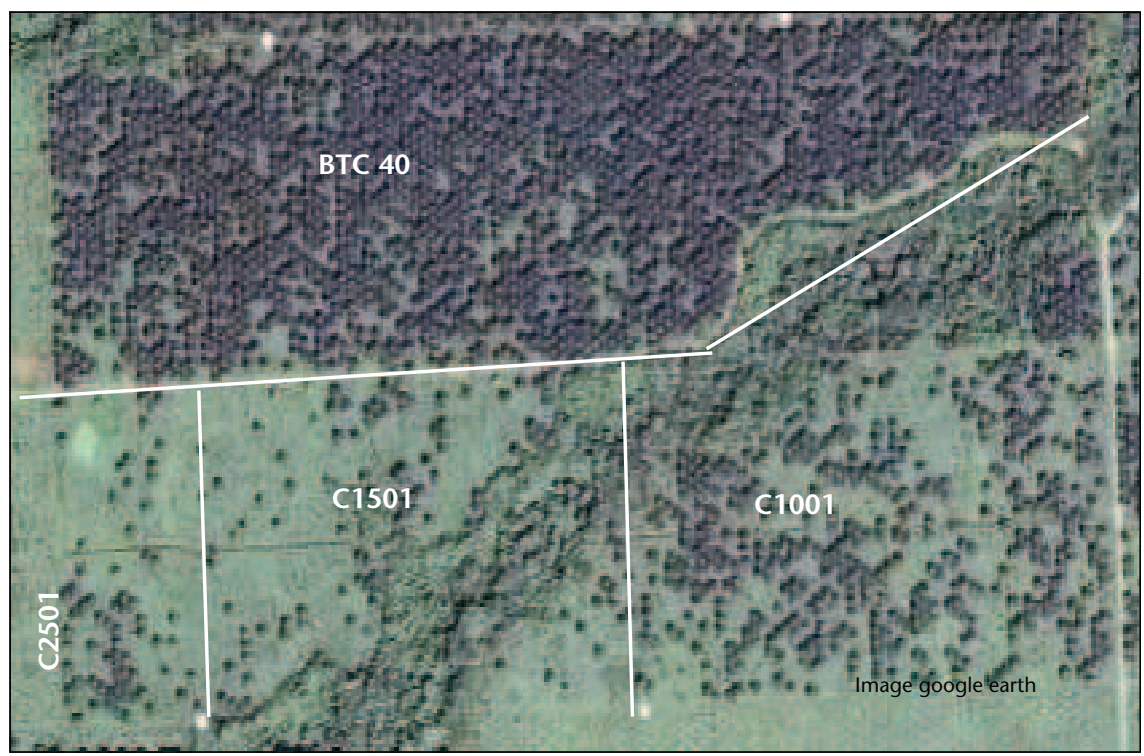

Figure 7. Comparaison du comportement au champ vis-à-vis de la PC d'un clone E. guineensis pur, BTC 40, par rapport à trois variétés E. guineensis dont une, C1001, représente l'origine dont il est issu. (Plantation de Shushufindi, PDE, Équateur). Le clonage par CIV permet de tirer parti de la variabilité intrafamille. saires, y compris la plantation de champs E. guineensis producteurs de pollen dans des zones non soumises à la maladie.

\section{Autres voies d'amélioration génétique du palmier à huile}

D'autres caractéristiques du palmier à huile pourraient être améliorées, chacune présente un intérêt et chacune s'inscrit bien dans des perspectives de développement durable. Nous pouvons les citer ici pour mémoire, mais sans les développer :

- les caractéristiques de l'huile de palme peuvent évoluer, surtout à travers l'usage de palmier $E$. oleifera, vers des teneurs élevées en acides gras insaturés ;

- le palmier à huile produit une huile naturellement très riche en vitamine $A$ (jusqu'à $3 \%$ ) et en vitamine $E$. Des sélections peuvent être envisagées sur ces points ;

- la valorisation des intrants est un point important que nous travaillons avec les agronomes et physiologistes;

- au-delà des problèmes de pollinisation de I'hybride, le palmier à huile évolue peu à peu vers une production d'un plus grand nombre d'inflorescences femelles : les fruits font le rendement. II faut cependant compenser cette féminité par la plantation simultanée d'arbres plus masculins. Le futur sera un palmier diöque.

Enfin, pour apporter de la souplesse dans l'organisation de la récolte, il est possible de sélectionner des palmiers à faible teneur en lipase et aux fruits indéhiscents. Cela permettrait d'espacer les tours de récolte et de mieux organiser l'usinage. En effet, la fréquence des tours de récolte a pour but de minimiser la proportion de fruits détachés (qu'il faut alors ramasser manuellement), et l'usinage quasi immédiat est nécessaire pour éviter une trop forte acidification liée à l'activité des lipases endogènes.

\section{Responsabilité sociale et entrepreneuriale}

C'est à plusieurs titres que PalmElit SAS met en œuvre sa stratégie en termes de Responsabilité sociale et entrepreneuriale (RSE) :

- comme toute entreprise nouvellement créée, PalmElit doit définir ses propres valeurs; les définir en relation avec le monde qui l'entoure est naturellement approprié ;

- PalmElit intervient comme acteur d'une filière à haute sensibilité sur les questions sociales et environnementales ;

- PalmElit se veut exemplaire en tant que sélectionneur et dans ses rapports avec les producteurs de semences; 
- PalmElit est aussi jugée à l'aune des actions de ses partenaires et clients planteurs; elle doit dans ce cadre s'efforcer de les amener à des comportements responsables;

- filiale du Cirad et de Sofiproteol, PalmElit hérite des valeurs de ses actionnaires (http:// www.cirad.fr/qui-sommes-nous/nos-valeurs et http://www.prolea.com/index.php?id=11129) et a reçu un cadrage clair de ses actionnaires pour établir un code de conduite.

Nous soulignerons quelques interrogations ou dilemmes auxquels nous avons dû répondre lors de l'élaboration de notre stratégie RSE.

\section{Risques auxquels PalmElit s'expose}

Contribuant, bon an mal an à la diffusion de 50 à 60 millions de semences, PalmElit et le Cirad mettent à la disposition des planteurs un matériel végétal qui suffit pour planter ou replanter jusqu'à 0,27 million d'hectares de palmiers chaque année. Sans règles de distribution, cela pourrait potentiellement placer PalmElit en position de complicité avec, par exemple, des actions de déforestation irresponsables ou de déplacement forcé de communautés.

Cela mettrait en cause la réputation non seulement de PalmElit, mais aussi celle du Cirad et de Sofiproteol. Même si les activités amont de la production sont peu regardées, PalmElit souhaite apporter sa contribution au développement $d$ 'une filière responsable.

PalmElit distribue des semences sous licence de marque qui est liée à une haute qualité du produit et des services associés. Des pratiques non éthiques de sa part affecteraient significativement cette image et la réputation de ses actionnaires et de ses principaux partenaires. Une difficulté majeure réside dans le fait que les pratiques non éthiques de ses clients (difficilement maîtrisables) auraient le même effet.

\section{Opportunités}

Un nombre continuellement croissant de producteurs s'engage dans la production d'huile durable et certifiée (www.rspo.org). La traçabilité est encore souvent dématérialisée à travers des certificats, mais la demande pour une traçabilité plus « pure » croît également et quelques producteurs s'organisent pour y répondre. Aujourd'hui, PalmElit travaille peu en Indonésie et en Malaisie et bien plus avec «le reste du monde ». Mais PalmElit a intérêt à promouvoir le développement d'une filière durable, moins contestée, et doit saisir l'opportunité d'amener autant de producteurs que possible dans cette démarche. Aujourd'hui, I'initiative RSPO et la certification qu'elle propose sont l'instrument principal sur lequel s'appuyer. De plus, PalmElit souhaite contribuer à améliorer la prise de conscience des petits producteurs, les aidant aussi à obtenir de meilleurs rendements et de meilleurs revenus tout en réduisant l'expansion de leur culture sur de nouvelles terres. Enfin, associer l'image d'un produit de haute qualité et le développement durable semble mutuellement bénéfique, et PalmElit trouve là une opportunité de démontrer ses compétences et ses convictions dans ces domaines.

\section{Aspirations de PalmElit}

PalmElit a rejoint la table ronde pour une huile de palme durable (RSPO) en 2009, s'engageant ainsi à promouvoir la durabilité, le respect de l'environnement, la protection de la biodiversité et le respect des droits de l'homme. Cet engagement se retrouve au cœur du métier de création variétale et nous avons souligné pourquoi dans les parties précédentes, mais nous aspirons également à favoriser des relations commerciales de long terme plutôt que de court terme avec l'ensemble de nos clients. L'approche doit être pragmatique et opérationnelle, basée sur une forte conviction que l'intérêt général doit être recherché sans toutefois compromettre le développement de notre compagnie.

\section{Valeurs}

Les valeurs de PalmElit ont été définies ainsi : - agir à long terme pour l'intérêt général ; - accompagner le développement du palmier à huile au profit du plus grand nombre de planteurs, en particulier pour les pays en développement ;

- les semences et les plants de PalmElit sont continuellement améliorés pour le bénéfice de toute la chaîne de production en réponse à ses besoins ;

- PalmElit développe ses activités en prenant en compte toutes les dimensions de la durabilité (économiques sociales et environnementales) ; - les partenariats de PalmElit sont transparents et équitables pour toutes les parties.

\section{Étendu des responsabilités de PalmElit}

PalmElit a peu d'impact direct sur la chaîne de production d'huile de palme, mais elle en a un plus important indirectement à travers ses clients en aval et ses partenaires en amont. Le souhait de PalmElit est de promouvoir auprès d'eux les bénéfices d'une huile de palme durable. Pour cela, sa sphère d'influence et sa sphère de contrôle doivent être précisées. Le souhait de PalmElit est d'accompagner ses partenaires et clients dans leur engagement de manière positive et sans exclusion, ce qui ne saurait être qu'un dernier recours.
On peut sans doute distinguer une continuité de relations de la plus faible à la plus forte :

- pas ou faible influence: ONG, petits planteurs, gouvernements (et, en particulier, leurs stratégies d'utilisation des terres et de maintien des forêts), consommateurs ;

- influence significative: partenaires de recherche et de production, prestataires, clients, actionnaires ;

- contrôle : employés, nouveaux actionnaires. Les clients, acheteurs de semences ou de plants, sont les plus nombreux dans la sphère $d^{\prime}$ 'influence de PalmElit. PalmElit n'a logiquement que peu d'influence sur leurs pratiques, mais se propose de les accompagner dans leur engagement de manière positive. Cependant, en dernier recours, un refus de vente est prévu en cas de manquements graves et reconnus aux principes de durabilité. À terme, si l'initiative RSPO devient une référence générale, PalmElit pourra exiger des grandes plantations I'adoption des principes et critères de RSPO et I'obtention d'une certification. PalmElit devra s'appuyer sur cette initiative dans la mesure où elle est aujourd'hui la plus complète et où elle se déploie rapidement. Pour les petits planteurs, des considérations spécifiques devront être appliquées de façon circonstanciée (selon les pays et surfaces en cause).

Bien évidemment, PalmElit et ses partenaires devront être exemplaires.

\section{Code de conduite}

La démarche exposée précédemment est traduite en un code de conduite qui fait le lien entre les valeurs exprimées et leur mise en œuvre pratique. II prend en compte les limites de la sphère de responsabilité de PalmElit, il est le document de référence pour ses propres opérations, mais également pour les partenaires.

Des objectifs chiffrés doivent être donnés. Pour l'exigence de certification, nous avons choisi de donner des dates, tout en étant parfaitement conscients que, si l'initiative RSPO ne se généralise pas comme espéré, ces échéances devront être repoussées.

Cependant, dès maintenant, une attitude claire peut être adoptée: vigilance sur les droits de l'homme, refus de liens avec les pays mis à l'index par I'Union Européenne, rejet de toute corruption, demande adressée aux clients planteurs d'être membres de RSPO, soutien de l'expansion de cette organisation, etc.

\section{Conclusion}

La durabilité est un concept large qui englobe des valeurs d'intérêt général. II n'est donc pas étonnant de retrouver cette notion de façon 
très transversale dans des domaines d'activité divers : nous avons donné ici plusieurs exemples dans lesquels la durabilité s'inscrit bien au cœur de l'action du sélectionneur. La génétique participe largement, mais certainement pas seule, à renforcer la durabilité des plantations $\mathrm{d}$ 'huile de palme : le rendement, la résistance aux maladies sont des exemples précis.

À travers l'exemple des hybrides interspécifiques résistants à la pourriture du cœur, nous avons montré comment la sélection pourrait, si on n'y remédie pas, avoir des conséquences négatives sur certains aspects sociaux du développement du palmier à huile.

Au-delà des aspects scientifiques et techniques, il est également possible de promouvoir la durabilité de la filière lors de la distribution des semences. L'influence d'un distributeur de semence est certes indirecte, mais notre réflexion montre qu'il est néanmoins possible $d^{\prime}$ agir, seuls et avec nos partenaires, en premier lieu pour réduire les risques auxquels notre entreprise, nos actionnaires et nos partenaires s'exposent dans le domaine d'une filière à haute sensibilité sur les questions sociales et environnementales. De façon plus positive, il est possible de promouvoir auprès des planteurs l'idée qu'il est aussi de leur intérêt de s'inscrire totalement dans la démarche de production d'une huile de palme durable. C'est certainement une opportunité à saisir qui contribuera à améliorer leurs rendements et leurs revenus tout en réduisant l'expansion de leur culture sur de nouvelles terres.

Sur la base de ces réflexions, il a été possible de définir des valeurs dans lesquelles quelques mots-clés ont été soulignés : long terme, intérêt général, équité et durabilité.

\section{RÉFÉRENCES}

Amblard P, Louise C, Zambrano J, et al. El programa de mejoramiento de la palma aceitera de PalmElit y de sus socios en Ecuador y Colombia. "Challenges in sustainable oil palm development". 2009. XVI Palm Oil International Conference, $23^{\text {rd }}$ to $25^{\text {th }}$ of September 2009, Cartagena de Indias, Colombia.

Beirnaert A, Vanderweyen R. Contribution à l'étude génétique et biométrique des variétés d'Elaeis guineensis Jacquin. Pub Inst Nat Etude agron Congo Belge Ser Sci $1941 ; 27: 1-101$.
Breton F, Miranti R, Lubis Z, et al. Ganoderma disease of the oil palm: hypothesis on natural infection and infection and implementation of an early artificial inoculation test to screen oil palm progenies for their level of resistance. XVI Palm Oil International Conference, $23^{\text {rd }}$ to $25^{\text {th }}$ of September 2009 Cartagena de Indias Colombia.

Cochard B, Noiret JM, Baudouin L, Flori A, Amblard P. Second cycle reciprocal recurrent selection in Oil Palm, Elaeis guineensis Jacq. Results of Deli x La Mé hybrids tests. Oléagineux 1993 ; 48 : 441-51.

Cochard B, Adon B, Kouamé KR, Durand-Gasselin T, Amblard P. Intérêts des semences commerciales améliorées de palmier à huile (Elaeis guineensis Jacq.). $\mathrm{OCL}$ $2001 ; 8: 654-8$.

Cochard B, Amblard P, Durand-Gasselin T. Oil palm genetic improvement and sustainable development. OCL $2005 ; 12$ : 141-7.

Davidson L. Management for efficiency, cost effective and productive oil palm plantations. In : Proc of 1991 Porim International Palm Oil Conference - Agriculture (Ed. By Basiron), 1993, 153-167. Palm Oil Res. Inst. Malaysia. Kuala Lumpur.

Durand-Gasselin T, Kouame Kouame R, Cochard B, Adon $B$, Amblard P. Diffusion variétale du palmier à huile (Elaeis guineensis Jacq.). OCL 2000 ; 7 : 203-6.

Durand-Gasselin $T$, de Franqueville $H$, Diabaté $S$, Cochard B, Adon B. Assessing and utilizing sources of resistance to Fusarium wilt in oil palm (Elaeis guineensis Jacq.) genetic resource. Paper presented at Int. Symp. "Oil palm genetic resources and utilization". 8-10 June, 2000, Malaysian Palm Oil Board, Kuala Lumpur.

Durand-Gasselin T, Cochard B, Amblard P, de Franqueville $H$. Un regard sur 40 ans d'amélioration génétique du palmier à huile (Elaeis guineensis) et son impact sur la filière. Le sélectionneur Français, Septembre 2002, ASF 1962-2002, 40 ans de sélection végétale 2002 : 133-48.

Durand-Gasselin T, de Franqueville H, Hayun Z, et al. Is it possible and reasonable to produce 10 tonnes of oil per hectare of oil palm. ISOPB, Medan, 6-9 October 2003.

Durand-Gasselin T, Asmady H, Flori A, et al. Possible sources of genetic resistance in oil palm (Elaeis guineensis Jacq.) to basal stem rot caused by Ganodermaboninense - Prospects for future breeding. Mycopathologia $2005 ; 159$ : 90-100.

Durand-Gasselin T, Corredor J, Sanz JI, de Franqueville $H, A m b l a r d ~ P$. Future vision of oil palm genetic improvement in Latin America. Several resolutions on cooperation in Colombia for planting material improvement. "Challenges in sustainable oil palm development". XVI Palm Oil International Conference, $23^{\text {rd }}$ to $25^{\text {th }}$ of September 2009, Cartagena de Indias, Colombia. de Franqueville $\mathrm{H}$, Renard JL. Improvement of oil palm vascular wilt tolerance. Results and development of the disease at the R. Michaux plantation. Oléagineux $1990 ; 45$ : 399-405.

de Franqueville $\mathrm{H}$, Asmady $\mathrm{H}$, Jacquemard JC, Hayun $Z$, Durand-Gasselin T. Indications on sources of oil palm (Elaeis guineensis Jacq.) genetic resistance and susceptibility to Ganoderma sp, the cause of basal stem rot. In: Proc 2001 Int. Palm Oil Congr. - Agriculture, 2001: 420-31. Malaysian Palm Oil Board, Kuala Lumpur.

de Franqueville $\mathrm{H}$. Oil palm bud rot in Latin America. Expl Agric $2003 ; 39$ : 225-40.

Gascon JP, de Berchoux C. Caractéristiques de la production d'Elaeis guineensis (Jacq.) de diverses origines et leurs croisements. Application à la sélection du palmier à huile. Oléagineux $1964 ; 19$ : 75-84.

Gascon JP, Jacquemard JC, Houssou M, Boutin D, Chaillard H, Kanga Fondjo F. La production de semences sélectionnées de palmier à huile Elaeis guineensis. Oléagineux $1981 ; 36$ : 476-86.

Gascon JP, Le Guen V, Nouy B, Asmady, Kamgo Fondjo F. Results of second cycle recurrent reciprocal selection trials on oil palm Elaeis guineensis Jacq. Oléagineux $1988 ; 43: 1-7$.

Hardon J), Corley RHV, Lee CH. Breeding and selecting the oil palm. In : Abott AJ, Atkin RK, eds. Improving vegetatively propagated crops. London : Academic Press, 1987 : 63-81.

Jannot C. Les déterminants historiques du commerce de I'huile de palme. Actes de la conférence internationale sur l'avenir des cultures pérennes. Yamoussoukro (Côte-d'Ivoire), 5 au 9 septembre 2001.

Lubis AA. History and origin of oil palm breeding material used in Marihat research station. ISOPB Workshop on dura and tenera population, Marihat, 1988.

Martinez G, Sarria GA, Torres GA, et al. Phytophthora $\mathrm{sp}$ es el responsable de las lesiones iniciales de la pudrición des cogollo (PC) de la palma aceite en Colombia. En memorias de la VIII Reunica Técnica National de Palma Aceite. 2008, Compensar, 22-24 Septiembre, Bogotá.

Renard JL, Noiret JM, Meunier J. Sources et gammes de résistance à la fusariose chez le palmier à huile Elaeis guineensis et Elaeis oleifera. Oléagineux 1980 ; 35 : 387-93.

Renard JL, Meunier J. Research for durable resistance to vascular wilt disease (Fusarium oxysporum f. sp. Elaeidis) of oil palm (Elaeis guineensis) in durable resistance in crops. In : Lamberto F, Waller JM, Van der Graaf NA, eds. Plenum Publishing Corporation, 1983. 